\title{
A proposal for the application of fractal geometry in describing the geometrical structures of water supply networks
}

\author{
D. Kowalski, B. Kowalska \& P. Suchorab \\ Department of Water Supply and Waste Water Disposal, \\ Lublin University of Technology, Poland
}

\begin{abstract}
The paper presents the problem of describing a water supply network as a complex geometrical structure. The authors suggest that many problems connected with the design and various aspects of exploitation of water supply networks may result from the lack of a universal language of their description. As a solution, the authors propose using the theory of fractal geometry. In the authors' opinion, it allows us to unify the description of branched as well as looped water networks, including their size and complexity degree. The practical application of that theory requires us to verify whether the geometrical structures of water supply networks meet the formal conditions of fractal sets. Such estimation has been done on the basis of three model networks with various geometrical structures. The considerations and analysis presented in the paper seem to give the affirmative answer to the question raised in the study. As a result of the work done the authors have elaborated the iterative formula which allows the description of the flat geometrical structures of branched and looped water networks. The formula is based on tree-like structures and several assumptions of their application.
\end{abstract}

Keywords: water supply, network, geometrical structures, description.

\section{Introduction}

One of the basic characteristics of water supply networks is their uniqueness. Practically, there are no two identical networks. That fact is a result of many factors which influence both the structure and transfer capacity of water distribution networks. This uniqueness requires the application of an individual 
approach to water supply networks at the stage of their designing as well as their exploitation. Since the beginning of modern water supply networks, universal methods of describing them have been searched for. Most attempts were directed towards a geometrical structure of networks. Based on that fact, a distinction is made between branched, looped and mixed water supply networks. This kind of description needed a supplement which would take into consideration the size and function of a water network. Therefore, there is also the division into local, communal, group, regional, etc. water supply networks. Currently, to fully describe a water network it is necessary to use a series of definitions connected with the existing classification. It is also essential in a description to resort to numbers, e.g. two-, four-, many-looped networks. That kind of approach does not allow us to unify the descriptions of every water network structure. The lack of standardisation causes problems at the stage of designing, optimisation of work conditions and monitoring of every water supply network. Each of these issues has to be considered separately as well as individually for every water supply network. It seems that further development in this discipline needs a new and universal language of description, which could be applied to every kind of water supply network.

From the point of view of their geometrical structure, water supply networks have a quasi chaotic character. Is it possible to distinguish a universal order and regularities occurring in this chaos? There is some hope in this regard due to fractal geometry, based on sets called fractals. Gleick [1] described fractal geometry as a kind of chaos boundary. Currently, the language of fractal geometry is successfully used to describe such a complex objects as a crack network of material subjected by outside loads [2, 3], clouds, trees, lines of horizon, etc. [4-6]. Is it then possible to consider water supply networks as sets which can be described in the language of fractal geometry? An answer to this question is given in this paper.

Despite the fact that the notion of fractals has been introduced over three decades ago, there is no commonly accepted definition of them. A lexicon of science and technology [7] defines the word fractal as a geometrical self-similar set (each part of the set is similar to the entirety in some scale. The word fractal comes from the Latin fractus - broken.

One of the first definitions of fractals was proposed by Mandelbrot [8], suggesting that the most appropriate is a definition in descriptive form. According to that, a fractal set is a set of parts similar to (reflecting) wholeness. The similarity may be exact, with precise copies of the entirety with successive changes of scales. What's more, the similarity may be statistical, where subsets are similar to the wholeness, but not identical with it. The quoted definition confirms an existence of scale invariability - an object looks similar at different scales of observation. An example of precise similarity is classic fractals, e.g. Cantor's set [9], Koch's curve [10] or Sierpiński's triangle [11]. Statistical similarity may be observed e.g. in a coast line, where successive magnifications reveal new peninsulas, bays, single rocks, but various at different scales [8].

The concept of self-similarity suggests that every element of a fractal set is created by different modifications of a basic element, e.g. by its magnification, 
rotation or translation [12]. Elements of a set created in this way become basic elements in another generation of modification. That kind of approach to the problem suggests that in order to construct new examples of fractals, a set of iterative methods may, or even should be applied. It is worth noticing that the iterative method creates a possibility of constructing fractals whose self-similar characteristics will be based on statistical or probabilistic dependences. Many authors have proved that fractals created in this method regularly reflect real objects better than classic fractals with exactly determined self-similarity $[13,14]$.

In the case of classic fractals the construction process is practically unlimited when it comes to the number of iterations. On the other hand, regarding natural object or objects built by humans it is different. For instance, by enlarging the magnification of a material's surface subjected to damaging loads, an ever thicker crack network can be observed. However, there is a line after crossing where further magnification will not be connected with the observed density. That line may be the size of molecules which forms a given material. These kinds of structures are sometimes called pre-fractals [14]. Nevertheless, many applications indicate that even in that case fractal geometry methods allow for a proper description of these structures.

However, the descriptive definition of fractals quoted above is imprecise due to its generality. Being aware of that, its creator completed it with a topological dimension, claiming that fractals are those self-similar sets whose Hausdorff's dimension [15] is given by a partial number [8].

The topological dimension is found by many authors as a central point of fractal geometry [12, 16, 17]. An implementation of Hausdorff's dimension postulated by Mandelbrot often becomes very difficult, especially in those sets where Hausdorff's dimension is greater than 1. As Zhou and others [18, 19] claim, this is the result not only of computational problems but also of the challenge posed by a full understanding of Hausdorff's dimension. Therefore, in practical applications other definitions of fractal dimensions (called covering dimensions) are used, such as box counting or Minkowski's dimension $[12,17]$.

\section{The research object}

The test study whether water supply networks may be considered as fractal sets was based on three example objects: a branched network (Fig. 1), a symmetrical one-looped network (Fig. 2) and a branched-looped mixed network (Fig. 3). In these considerations such aspects as water partition, hydraulic pipe load or water pressure level were omitted. The authors focused only on the geometrical structure of the given networks. In the cases of looped and mixed networks the problem of diameter diversity was also omitted. Additionally, in order to define a fractal dimension, two of the chosen networks were written in the rectangular coordinate system $(\mathrm{X}, \mathrm{Y})$. As a basic unit of their size, the length of these networks' projections on the $\mathrm{X}$-axis were assumed. 


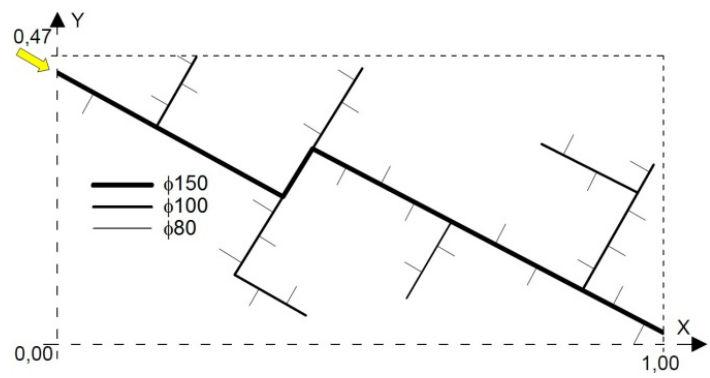

Figure 1: An example of a branched water supply network. The source of water supply is marked by an arrow.
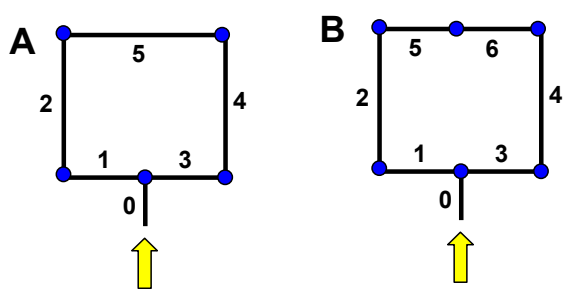

Figure 2: A symmetrical one-looped water supply network, with a differentiation of changing direction and branching nodes. A without the distinction of a node which divides a source segment opposite to water supply, B - with a distinction of that node.

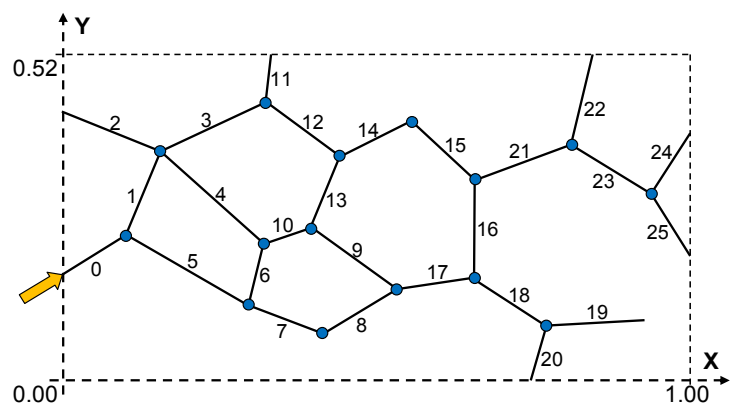

Figure 3: An example of a 4-looped network with branches and directionchanging and branching nodes. $1,2 \ldots .25$ - numbers of particular segments.

\section{The research methodology}

Taking into consideration the fractal set definition given previously by Mandelbrot and others, an attempt has been made to see whether selected water supply networks fulfil the definition's demands. In order to do that, the following questions should have been answered: 
- is the considered set too irregular to be described by the language of traditional geometry?

- $\quad$ is there a scale hierarchy in it? does each successive magnification of scale reveal an existence of consecutive details?

- is that set not given by a formula but a recurrent dependence? does each successive iteration of this dependence lead to a specialisation or magnification of this set?

- does the set have one or many self-similar characteristics?

- is a topological dimension of that set not an integer?

The answer to the first question was sidetracked by the issue whether the water pipe structure of the networks considered can be described by a mathematical function. In Figures 1 and 3 the networks were written in the Cartesian coordinate system X, Y. A domain of the contained set includes $\mathrm{X}<0$, $1>$, whereas values remain in the range of $Y<0,0,0,47\rangle$ and $\langle 0,0,0,52>$. A function, defined in set $X \subset R$ with values in set $Y \subset R$, is an assignment which to every element $x \in X$ assigns exactly one element $y \in Y$ [20]. Therefore, the networks considered are not functions in the sense of the above definition. In both cases shown in Figures 1 and 3, to one argument $\mathrm{x} \in \mathrm{X}$ it is possible to assign several values of $y \in Y$. Only the division of every considered network into component segments will make it possible to describe the examined structure by compounding many functions, where each corresponds to a chosen segment. Therefore, in view of the aspect investigated, it seems than the answer to the first question is affirmative.

The question of the existence of a scale hierarchy in the selected water supply networks was based on the network shown in Figure 1 (the only one with pipe dimension differentiation). It was assumed that the examined network is observed under the different magnifications. At the beginning, a magnification allows an observation only of objects greater or equal to $150 \mathrm{~mm}$ (the biggest of the used dimensions). The next resolution of a picture is $100 \mathrm{~mm}$, and then $80 \mathrm{~mm}$. An affirmative answer to the question of scale hierarchy suggests that the picture obtained should have more and more details. This kind of situation should depend on the existence of dimension differentiation, which in water supply networks is a principle. According to that, the problem in question was treated as analogical and skipped in the cases shown in Figures 2 and 3.

The questions of a recurrent dependence and the existence of self-similar characteristics in the networks examined should, in the authors' opinion, be considered together.

A recurrent dependence defines a kind of self-similarity existing in the examined sets. Many authors $[12,21,22]$ claim that the issue of defining a recurrent dependence is a key to a fractal set description. As a initial hypothesis, it was established that a geometrical structure of water supply networks can be presented by a tree-like (dendritic) structure. Successive elements of that structure are created by copying, moving, and rotating a basic segment in each iteration. The basic description of the development of that kind of structures was given by Prusinkiewicz and Lindenmayer [23]. They proposed a formalism, first used to describe a plant's growth. This formalism was later used in computer 
modelling of many other issues of fractal geometry. Currently, it is known as parallel rewriting systems or L-systems [12]. The formalism requires a beginning value, called in that notation an axiom, and a formula of consecutive transformation of this value. In the case of a tree-like structure, assumed by the authors, this will look as follows:

$$
\begin{array}{r}
\text { axiom: } L_{0} \text { - beginning segment, } \\
\text { formula of transformation: } L_{i+1} \rightarrow\left\{\begin{array}{l}
a \cdot L_{i}, \alpha^{\prime} \\
b \cdot L_{i}, \alpha^{\prime \prime} \\
c \cdot L_{i}, \alpha^{\prime \prime \prime}
\end{array}\right.
\end{array}
$$

The given formula responds to iterative transformations of a previous segment $L_{i}$ to a consecutive one $L_{i+1}$, where $a, b, c$ are the length parameters of the newly created segments, and $\alpha^{\prime}, \alpha^{\prime \prime}, \alpha^{\prime \prime}$ - angles describing the localisation of the newly created segments in accordance with the previous segment. There is also a rule that new segments connect with the end of a previous ones. Every successive transformation of a basic segment is a modification, which in fractal geometry is often called a bifurcation.

Additionally, in describing the water supply networks shown in Figures 1-3, the following assumptions have been made:

- $\quad$ parameters $a, b, c$ and angles $\alpha^{\prime}, \alpha^{\prime \prime}, \alpha^{\prime \prime}$ may assume random values, which in practice depends on the shape of the street where water pipes are installed,

- when one of the parameters $a, b, c$ equals zero, this means that a particular segment is excluded from further transformation,

- when different branch segments of a tree-line structure cross, a new node is created, which can be a starting point to new transformations. In that case, the basic segment for further transformations is one of the segments which was directly crossing.

Based on the formula of transformation (1) and assuming $a, b, c$ as a equal and invariable values and $\alpha^{\prime}=-\alpha^{\prime \prime}$ and $\alpha^{\prime \prime}=0$, a tree-line symmetric structure is drawn, whose construction is shown in Figure 4.

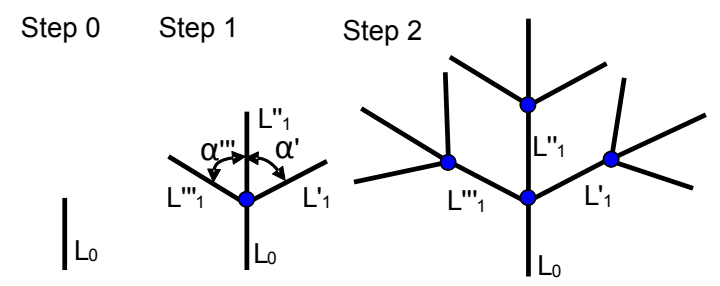

Figure 4: Consecutive steps of creating a symmetric tree-line structure.

Supposing that, in consecutive transformations, parameters $a, b, c$ assume random values from the set of real numbers $<0,+\infty)$, that angles $\alpha^{\prime}, \alpha^{\prime \prime}, \alpha^{\prime \prime}$ assume random values from a set $(-\pi, \pi)$ and that the assumption by one of the $a$, $b, c$ parameters of the value of zero excludes that particular segment from further transformations, an unsymmetrical tree-line structure may be drawn, whose example is shown in Figure 5. 
Using the scheme of constructing tree-line structures given in Figures 4 and 5, a hierarchic dependence of its segments can be shown (Figure 6). This comes from a river's classification and is mainly used to describe the structure of blood vessels [24], but also by Bejan [21] to prove the existence of self-similar characteristics in tree-line structures.
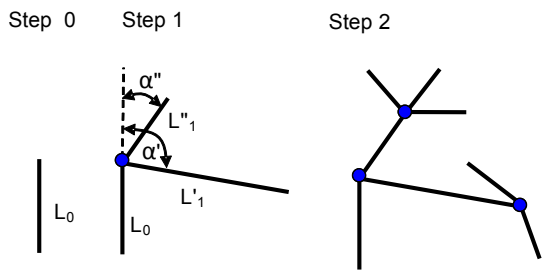

Figure 5: An example of a unsymmetrical tree-line structure's construction with an assumption of random formula (1) transformation's parameters

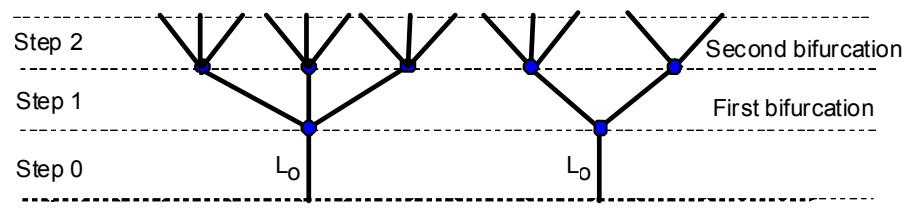

Figure 6: A scheme of hierarchical dependence of segments in tree-line structures. On the left is the structure from Fig. 4, on the right from Fig. 5.

To define the fractal dimension of networks shown in Figures 1 and 3 the box counting method was used. Its detailed description is given mainly by $[12,17]$. In that method a given geometrical set (in the examined networks enclosed in a surface) is covered by squares. Then, a logarithmic dependence between their size $(\delta)$ and an indispensable number required to cover that set $(N)$ is estimated. Searching for the fractal dimension is defined as equal to a directional coefficient of a regression line:

$$
\log (N)=a \cdot \log (\delta)+b
$$

In cases of fractal sets, this dimension should not be given by an integer.

\section{Results and discussion}

Testing whether water supply networks fulfil the formal requirements of a fractal set was started with a branched network shown in Figure 1. By changing the observation's scale of a conventional "microscope" to various resolutions, the following cycle of figures may be observed (Figure 7). 


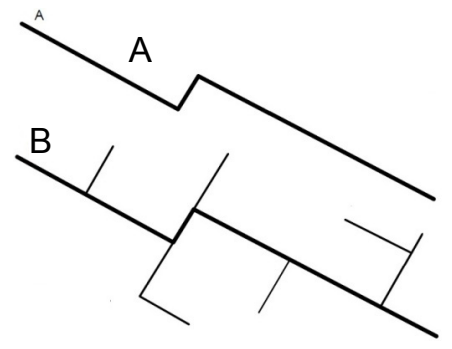

Figure 7: An examined network with the resolution of $\mathrm{A}-150 \mathrm{~mm}$ and $\mathrm{B}-100 \mathrm{~mm}$.

Comparing the pictures shown in Figures 1 and 7, it can be noticed that depending on the resolution used not only the amount of details observed is changing, but also the size of the picture, in this case measured along axis Y. This kind of situation corresponds with observing a crack network in material subjected to damaging load, described in the works of Mandelbrot et al. [2] and Dauskardt et al. [3]. Following their argument, it may be said that the examined water supply network does have the fractal set features of the existing observation's scale hierarchy. However, it should be remembered that increasing the picture's resolution to the value below a minimum dimension used will not change the network's picture. The examined set may thus be called pre-fractal in this respect.

It was then tested whether the assumed initial hypothesis (see Formula (1)), can be used to describe the structure of an examined network. To that end, a given network was divided into line segments, whose borders were changes in flow direction, their branches and free endings. A hierarchical dependence graph of chosen segments was drawn for that network (Figure 8).

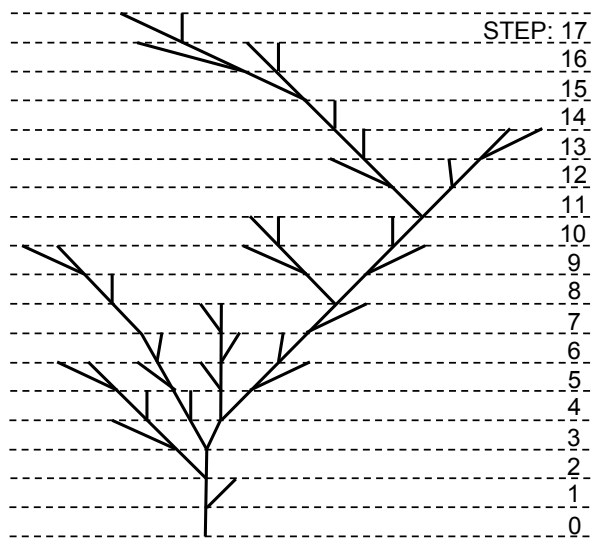

Figure 8: The hierarchical dependence of an examined network's pipes from Fig. 1, in keeping with the postulated description given by a treelike structure. 
The above graph shows that the analysed network can be built by 17 consecutive transformations, realised in accordance with Formula (1). That suggests the possibility of accepting the initial hypothesis as a correct one.

The analysis of the examined network was completed with its fractal dimension, calculated by the box counting method. In order to do that, a logarithmic graph showing a dependence between square size and the indispensable number required to cover that examined set was drawn (Figure 9).

The directional coefficient of the regression line shown in the figure is estimated as a fractal dimension value, and in that particular case is equal to 1.343. Therefore, it is not given by an integer.

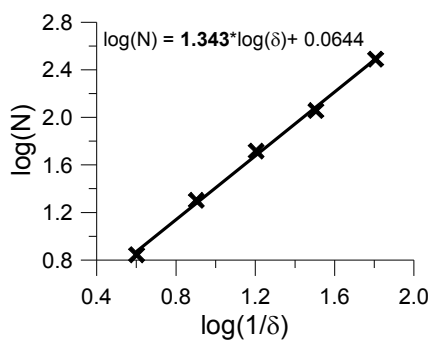

Figure 9: A logarithmic graph of the number of squares $(\mathrm{N})$ required to cover an examined water supply network in relation to the square's side size $(\delta)$.

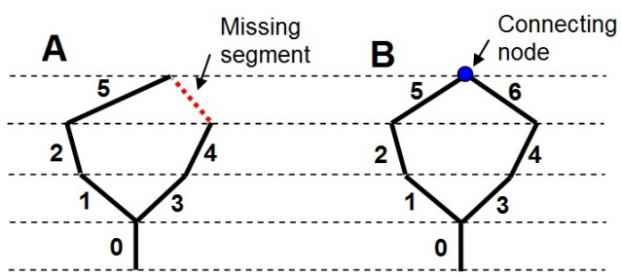

Figure 10: Hierarchical connections of the consecutive network segments shown in Figure 2.

In previous considerations, the branched network was analysed. Because this kind of network is only one of many classes of water supply networks, the authors decided to check whether looped networks can also be described by the language of fractal geometry. Among the emphasised conditions which fractal sets fulfil, the most important seems to be the one checking the existence, and if so the type, of similarity in looped networks. Considerations started with onelooped symmetrical network (shown in Figure 2). Similarly to a previous example, it was done by drawing a graph showing the hierarchical dependence of pipe segments forming a network (Figure 10).

Figure 10A shows the impossibility of closing the loop while fulfilling the previously accepted assumptions. Irrespective of whether segment 5 is led from segment 2 or 4 , the created structure does not close a loop. Having made an additional connecting node (Figure 10B), that kind of closing was possible. This 
means that in order to explain a looped structure construction in terms of treelike structures a creation of additional connecting nodes may be needed.

Water supply networks are rarely created by single, symmetrical loops. This being so, a test was run to see whether an accepted initial hypothesis explains the creation of more complex network structures. In order to do that, the structure of the water supply network shown in Figure 3 was considered.

Similar to previous examples, in order to confirm or deny the initial hypothesis about the possibility of generating looped structures by tree-line elements, a graph of the hierarchical dependence of particular segments was drawn (Figure 11).

The structure of the water supply network considered was created by 8 consecutive transformations of a basic segment. The construction of that structure needed the creation of two connecting nodes (Sections 9 and 13), which was accordance with previous assumptions (Figure 10).

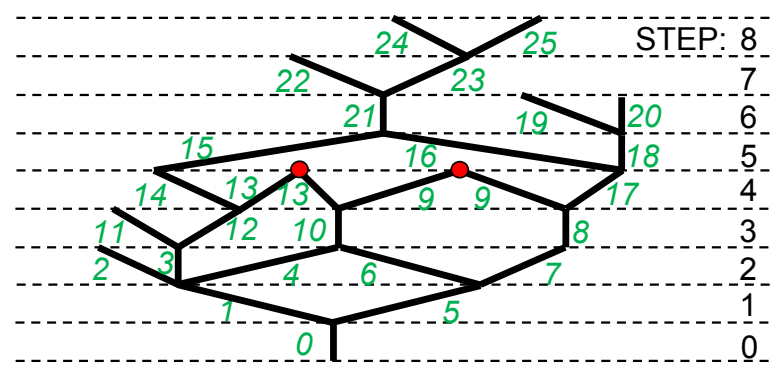

Figure 11: Hierarchical consecutive segment connections of the network shown in Fig. 3. 0,1,2,..,25 - number of section, - connecting node.

In order to complete the considerations about the formal demands of fractal sets, by analogy to the case of the branched network, the fractal dimension of the network shown in Figure 3 was calculated. This dimension was 1.326. Similarly to the previous case, the value of this dimension is not in the integer set.

It would be interesting to see if many-looped networks, supplied from many sides, might also be described by tree-like structures. These considerations were based on the network from the previous example, however its supply places were assumed from three sides (Figure 12). Here, too, the assumptions were as before.

In the case of the network shown in Figure 12, an attempt at creating a hierarchical connections graph of selected segments did not bring any satisfying results. The result obtained was quite unclear. Thus it was decided to show this dependence by the consecutive steps of the network's creation (Figure 13). Step 0 consisted only of the nodes shown in Figure 12 and the basic segments supplying the network with water. In Step 1 it was necessary to add a new connecting node "A", similarly to Figure 10B. In subsequent steps three more connecting nodes were added (Step 2 - "B", Step 3 - “C”, Step 4 - “D”).

The considered network supplied from many sides was created in 5 consecutive transformation steps of basic supplying segments. It is worth 
noticing that in the case of single supply, nine steps were required. The addition of four extra connecting nodes caused the possibility of describing the considered network by hierarchical connected tree-line structures. As in the previous example, this assumption does not exclude the legitimacy acceptance of the initial hypothesis.

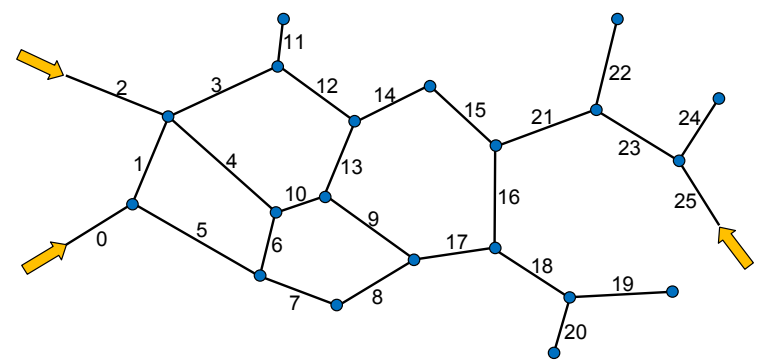

Figure 12: Example of network supplied from many sides. Places of water supplies are marked by arrows; $0,1,2 \ldots, 25$ are numbers of tagged segments.

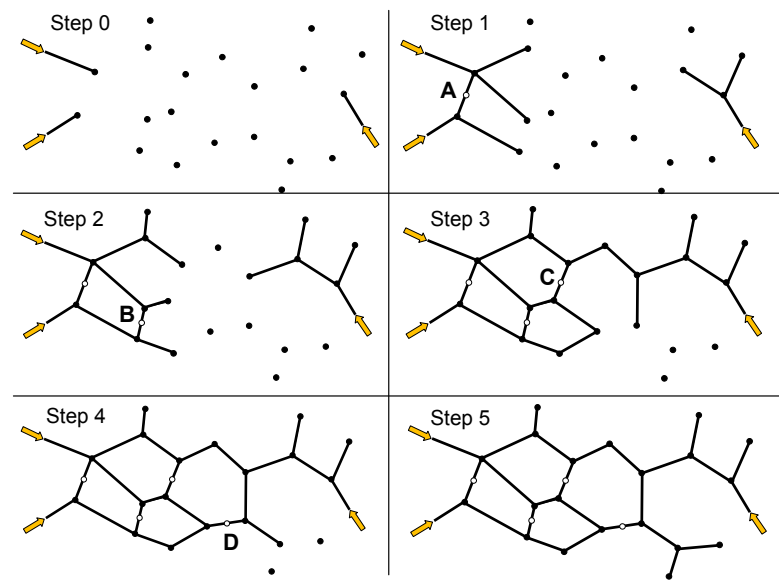

Figure 13: Consecutive creation steps of the examined water supply network by tree line structures; A, B, C, D-additional connecting nodes.

\section{Summary}

Following the argument in this paper, it can be said that the examined branched, looped and mixed water supply networks do have the character of pre-fractals with statistical self-similarity. During the analysis, the existence of scale hierarchy in water supply networks was confirmed. It was also shown that the 
structure of these networks cannot be described by single formulas. However, it is possible to achieve that structure by consecutive transformations, according to the formula given in the paper. Thus the assumed initial hypothesis about the possibility of describing both branched and looped networks by tree structures was confirmed. It should also be mentioned that the calculated fractal dimensions of examined networks were not integers. All of the above suggests that the water supply networks analysed may by described in the language of fractal geometry.

\section{Acknowledgement}

This paper was financed by statutory activity of the Faculty of Environmental Engineering, Lublin University of Technology.

\section{References}

[1] Gleick, J. Chaos. Making a New Science. Penguin Books, 1988.

[2] Mandelbrot, B.B., Passoja, D.E., Paullay, A.J., Fractal character of fracture surfaces of metals. Nature, 308(5961), pp. 721-722, 1984.

[3] Dauskardt, R.H., Haubensak, F., Ritchie, R.O. On the interpretation of the fractal character of fracture surfaces. Acta Metall. Mater., 38(2), pp. 143159, 1990.

[4] Mandelbrot, B.B., Van Ness, J.W., Fractional Brownian motions, fractional noises and applications. SIAM Rev., 10(40), pp. 422-437, 1968.

[5] Mandelbrot, B.B., Stochastic models for the earth's relief, the shape and fractal dimension of coastlines, and the number area rule for islands. Proceedings of the NAS of the USA, 72(10), pp. 2825-2828, 1975.

[6] Mandelbrot, B.B., On the geometry of homogeneous turbulence, with stress on the fractal dimension of isosurfaces of scalars. Journal of Fluid Mechanics, 72(2), pp. 401-416, 1975.

[7] Iwańska, J., Martin, M., Romkowska, E., Siemeniuk, B., Yopulos, A., Werner, M., (ed.), Leksykon naukowo-techniczny. WNT, Warszawa, 2001.

[8] Mandelbrot, B.B., The Fractal Geometry of Nature. New York: W. H. Freeman and Co., 1982.

[9] Cantor, G., Über unendliche, lineare Punktmannigfaltigkeiten V, Math. An., 21, pp. 545-591, 1883.

[10] Koch, H., Une méthode géométrique élémentaire pour l'étudé de certaines questions de la théorie des courbes planes. Act. Math, 30, pp. 145-174, 1906.

[11] Sierpiński, W., Sur une Courbet cantorienne qui contient une image biunivoquet et continua detoute Courbet donnée. C.R. Acad. Paris, 162, pp. 629-632, 1916.

[12] Peitgen, H.O., Jürgens, H., Saupe, D., Chaos and Fractals: New Frontiers of Science. Springer, New York, 1992.

[13] Neil, G., Curtis, K.M., Shape recognition using fractal geometry. Pattern Recognition, 30 (12), pp. 1957-1969, 1997. 
[14] Pentland, A.P., Fractal-based descriptions of natural scenes. IEEE Trans. Pattern Annal Machine Intell. PAMI-6, pp. 661-674, 1984.

[15] Hausdorff, F., Dimension und äussers Mass. Math. Annalen, 79, pp. 157179, 1919.

[16] Falconer, K.J., Fractal Geometry: Mathematical Foundations and Applications. John Wiley, Chichester, 1990.

[17] Kudrewicz, J., Fraktale i chaos. WNT, Warszawa, 2007.

[18] Zhou, Z., Feng, L., Twelve open problems on the exact value of the Hausdorff measure and on topological entropy: a brief survey of recent results. Nonlinearity, 17, pp. 493-502, 2004.

[19] Zhou, G., Lam, N., S-N., A comparison of fractal dimension estimators based on multiple surface generation algorithms. Computers \& Geosciences, 31, pp. 1260-1269, 2005.

[20] Gewert, M., Skoczylas, Z., Analiza matematyczna 1. GiS, Wrocław, 2003.

[21] Bejan, A., Shape and Structure, from Engineering to Nature. Cambridge University Press, Cambridge, UK, 2000.

[22] Gabryś, E., Rybaczek, M., Kędzia, A., Blood flow simulation through fractal models of circulatory system. Chaos, Solitons and Fractals, 27, pp. $1-7,2006$.

[23] Prusinkiewicz, P., Lindenmayer, A., The algorithmic Beauty of Plants. Springer Verlag, New York, 1990.

[24] Bavel, E., Spaan, J.A.E., Branching patterns in the porcine coronary arterial tree estimation of flow heterogeneity. Circ. Res., 71, pp. 1200$1212,1992$. 